\title{
COMPLICAÇÕES EM PACIENTES RENAIS CRÔNICOS SUBMETIDOS À HEMODIÁLISE*
}

\author{
Jéssica Dantas de Sá Tinôcoํㅜ, Maria das Graças Mariano Nunes de Paiva², Kadyjina Daiane Batista Lúcioº \\ Raissa Lopes Pinheiro ${ }^{4}$, Beatriz Medeiros de Macedo ${ }^{3}$, Ana Luisa Brandão de Carvalho Lira ${ }^{5}$
}

\begin{abstract}
RESUMO: Objetivou identificar as complicações em pacientes renais crônicos submetidos à hemodiálise e correlacioná-las aos fatores sociodemográficos e clínicos. Estudo transversal, com 200 pacientes em uma clínica de nefrologia no Nordeste do Brasil. Utilizou-se um formulário para a coleta de dados, que ocorreu durante março e abril de 2015. Na análise inferencial, foram aplicados testes de Qui-Quadrado e Exato de Fisher, e U de Mann-Whitney, na associação entre variáveis nominais e numéricas. As associações estatísticas foram: hipotensão com idade, sexo e ganho de peso interdialítico; calafrios com sexo e ganho de peso interdialítico; vômito com sexo, sítio de diálise e KT/V; cefaleia com sexo e idade; tontura com anos de estudo, religião e ganho de peso interdialítico; arritmia e idade; diarreia e tempo de hemodiálise; dor abdominal e ganho de peso interdialítico; sudorese e idade. Conclui-se que complicações durante a hemodiálise podem sofrer influência de fatores sociodemográficos e clínicos.
\end{abstract}

DESCRITORES: Enfermagem; Diálise renal; Complicações; Insuficiência renal; Perfil de saúde.

\section{COMPLICATIONS IN PATIENTS WITH CHRONIC RENAL FAILURE UNDERGOING HEMODIALYSIS*}

\begin{abstract}
The present study aimed to identify complications in patients with chronic renal failure undergoing hemodialysis and correlate them to socio-demographic and clinical factors. Cross-sectional study with 200 patients conducted in a nephrology clinic in the Northeast of Brazil. Data was collected in March and April 2015 through a structure questionnaire completed by the participants. Chi-square and Fisher's Exact tests were performed in inferential analysis, and Mann-Whitney U test was used to assess the relationship between categorical (nominal) and numerical variables. The statistically significant associations were hypotension with age, gender and interdialytic weight gain; chills with gender and interdialytic weight gain; vomiting with gender, dialysis site and $\mathrm{Kt} / \mathrm{V}$; headache with gender and age; dizziness with years of schooling, religious belief and interdialytic weight gain; arrhythmia and age; diarrhea and length of time on dialysis treatment; abdominal pain and interdialytic weight gain; sweating and age. It is concluded that complications during hemodialysis can be affected by socio-demographic and clinical factors.
\end{abstract}

DESCRIPTORS: Nursing; Renal dialysis; Complications; Renal failure; Health profile.

\section{COMPLICACIONES EN PACIENTES RENALES CRÓNICOS SOMETIDOS A HEMODIÁLISIS}

RESUMEN: Se objetivó identificar complicaciones en pacientes renales crónicos sometidos a hemodiálisis y correlacionarlos a factores sociodemográficos y clínicos. Estudio transversal, con 200 pacientes en clínica de nefrología del Nordeste de Brasil. Se aplicó formulario para recolección de datos, realizada durante marzo y abril de 2015. Para análisis inferencial fueron aplicados tests de Chi-cuadrado y Exacto de Fisher, y U de Mann-Whitney para asociación entre variables nominales y numéricas. Las asociaciones estadísticas fueron: hipotensión con edad, sexo e incremento de peso interdialítico; escalofríos con sexo e incremento de peso interdialítico; vómitos con sexo, sitio de la diálisis y KT/V; cefalea con sexo y edad; mareos con años de escolarización, religión e incremento de peso interdialítico; arritmia y edad; diarrea y tiempo de hemodiálisis; dolor abdominal e incremento de peso interdialítico; sudoración y edad. Se concluye en que las complicaciones durante la hemodiálisis pueden resultar influidas por factores sociodemográficos y clínicos.

DESCRIPTORES: Enfermería; Diálisis Renal; Complicaciones; Insuficiencia Renal; Perfil de Salud.

*Artigo extraído da dissertação intitulada "Validação clínica do diagnóstico de enfermagem proteção ineficaz em pacientes submetidos à hemodiálise". Universidade Federal do Rio Grande do Norte, 2015.

'Enfermeira. Mestre em Enfermagem. Docente de Enfermagem da Universidade Federal do Rio Grande do Norte. Natal, RN, Brasil.

${ }^{2}$ Enfermeira. Mestre em Enfermagem. Universidade Federal do Rio Grande do Norte. Natal, RN, Brasil.

${ }^{3}$ Enfermeira. Mestranda em Enfermagem. Universidade Federal do Rio Grande do Norte. Natal, RN, Brasil.

${ }^{4}$ Enfermeira. Preceptora do ensino técnico em enfermagem da Universidade Potiguar. Natal, RN, Brasil.

${ }^{5}$ Enfermeira. Doutora em Enfermagem. Docente da Pós-Graduação em Enfermagem da Universidade Federal do Rio Grande do Norte. Natal, RN, Brasil. 


\section{- INTRODUÇÃO}

A Doença Renal em Estágio Terminal (DRET) é caracterizada pela perda progressiva e irreversível das funções renais, resultando em distúrbios metabólicos graves ${ }^{(1)}$. A hemodiálise (HD) é o tratamento de substituição renal de primeira escolha mundial, e o Brasil acompanha essa realidade, em que $90 \%$ dos pacientes com DRET são submetidos à $\mathrm{HD}^{(2)}$.

A HD, apesar de proporcionar benefícios aos pacientes, gera inúmeras complicações relacionadas à excreção de solutos urêmicos, água e eletrólitos, à resposta do paciente e ao próprio processo de diálise. Essas complicações aumentam a morbidade e mortalidade dos pacientes renais em hemodiálise ${ }^{(3-4)}$.

A literatura ${ }^{(5-6)}$ relata que as principais complicações são: câimbra muscular, prurido e dor de cabeça. Afirma ainda que as complicações com prevalências menores são: dor abdominal, hipotensão, hipertensão, vômito, ganho de peso em curto período, constipação(6).

Percebe-se que existem variações na literatura relativas à prevalência das principais complicações, caracterizando a relevância de mensurá-las com maior precisão e levando em conta o contexto no qual esse paciente se encontra. Assim, torna-se necessário desenvolver estudos no intuito de identificar a prevalência dessas complicações, bem como relacioná-las aos fatores sociodemográficos e clínicos.

Ademais, entender as complicações junto às características sociodemográficas e clínicas nas quais estão inseridas implica maior atuação dos profissionais de saúde, principalmente do enfermeiro, o qual, com base nessa análise, considerará os aspectos sociais do paciente no planejamento de cuidados e de medidas preventivas. Autores ${ }^{(7)}$ reforçam a relevância de tal compreensão, no sentido de direcionar as ações de saúde à clientela com características de maior vulnerabilidade, contribuindo deste modo com a qualidade de vida e na redução de custos hospitalares.

Destarte, questiona-se: Quais são as complicações presentes nos pacientes renais crônicos em hemodiálise? Essas complicações sofrem influência dos fatores sociodemográficos e clínicos? Partindo desse questionamento, a presente pesquisa tem por objetivo identificar as complicações em pacientes renais crônicos submetidos à hemodiálise e correlacioná-las aos fatores sociodemográficos e clínicos.

\section{- MÉTODO}

Estudo do tipo transversal, realizado em uma clínica de referência em nefrologia no Nordeste do Brasil. A amostragem se deu por conveniência, de forma consecutiva, nos meses de março e abril de 2015.

A população se constituiu de 300 pacientes em tratamento hemodialítico na referida clínica. A amostra foi calculada a partir da fórmula para população finita, considerando o nível de confiança de $95 \%(Z a=1,96)$, o erro amostral de $5 \%$, a população de 300 pacientes e o valor conservador de $50 \%$ da prevalência. Resultou em uma amostra de 168, que foi arredondada para 200 indivíduos.

Os critérios de inclusão foram: pacientes a partir de 18 anos em tratamento hemodialítico na unidade de diálise referida. Os critérios de exclusão foram: indivíduos com dificuldade de comunicação verbal que impossibilitasse a coleta de dados e desorientados em relação ao tempo e espaço.

Utilizou-se como instrumento coleta de dados um formulário estruturado, contendo perguntas sobre os dados sociodemográficos, clínicos e as complicações vivenciadas pela clientela investigada durante a hemodiálise. O formulário foi submetido à validação de aparência e conteúdo por seis enfermeiros com estudos na área de enfermagem em nefrologia. As sugestões realizadas pelos enfermeiros foram incorporadas ao instrumento final.

Realizou-se o pré-teste, com a aplicação do formulário em 20 pacientes renais crônicos submetidos à hemodiálise. Não houve necessidade de alteração no instrumento. Dessa forma, os participantes do pré-teste foram incluídos na amostra do estudo.

Os dados foram coletados por pesquisadores previamente treinados, os quais conduziram a entrevista com o paciente, enquanto estes eram submetidos ao procedimento hemodialítico. 
Na organização e análise, construiu-se um banco de dados, no qual foram registrados os dados sociodemográficos, clínicos e as complicações identificadas. Para a análise dos dados utilizou-se o programa estatístico IBM SPSS Statistic ${ }^{\circledast}$, para gerar frequência absoluta e relativa, bem como medidas de tendência central e dispersão. A normalidade foi identificada pelo teste de Kolmogorov Smirnov. Para a análise inferencial e associação entre as variáveis nominais, foram aplicados os testes de QuiQuadrado e Exato de Fisher, e U de Mann-Whitney, na associação entre variáveis nominais e numéricas. O nível de significância adotado foi de $5 \%(p<0,05)$.

Foi aprovado pelo Comitê de Ética em Pesquisa da instituição responsável, sob o parecer número 387.837.

\section{RESULTADOS}

Dos pacientes entrevistados, 102 (51\%) eram do sexo feminino, viviam com companheiro 107 (53,5\%), eram praticantes de religião $173(86,5 \%)$, procedentes do interior do estado 108 (54\%) e apresentaram média de idade de 55 anos. O tempo médio de estudo foi de 8,69 anos e a renda familiar, de 3,76 salários mínimos.

No que se refere aos dados clínicos, os pacientes apresentaram tempo de diagnóstico de doença renal crônica de 7,9 anos e 5,6 anos de tratamento hemodialítico. Dos pacientes entrevistados, 150 (75\%) utilizavam fístula arteriovenosa (FAV) como sítio para a hemodiálise. Os participantes apresentaram média de ganho de peso interdialítico de 1,92 kg e KT/V (método que avalia a qualidade da diálise do paciente) de 1,21.

As principais complicações relatadas pelos pacientes durante o período hemodialítico foram: câimbras $(149 ; 74,5 \%)$, hipotensão $(141 ; 70,5 \%)$, calafrios $(104 ; 52 \%)$, vômitos $(77 ; 38,5 \%)$, cefaleia $(71$; $35,5 \%)$, tontura $(70 ; 35 \%)$, hipertensão $(48 ; 24 \%)$ e arritmia $(40 ; 20 \%)$. Complicações como náuseas, convulsões, diarreia e dor abdominal foram frequentes em apenas dois pacientes (1\%) da amostra estudada. Sono, agonia, sudorese, fraqueza, falta de ar, dormência, dores no corpo e tremores representaram $0,5 \%$ das complicações.

A Tabela 1 mostra a associação entre as complicações apresentadas pelos pacientes renais crônicos durante a hemodiálise e os dados sociodemográficos/clínicos.

Tabela 1 - Análise de associação entre dados sociodemográficos, clínicos e as complicações durante a hemodiálise em pacientes renais crônicos. Natal, RN, Brasil, 2017 (continua)

\begin{tabular}{|c|c|c|c|c|c|c|c|c|c|c|c|c|}
\hline Complicações & Sexo & Idade & $\begin{array}{c}\text { Estado } \\
\text { Civil } \\
\end{array}$ & Renda & Estudo & Procedência & Religião & $\begin{array}{c}\text { Tempo } \\
\text { DRCt }\end{array}$ & $\begin{array}{c}\text { Tempo } \\
\text { HD }\end{array}$ & $\begin{array}{c}\text { Sítio } \\
\text { Diálise } \\
\end{array}$ & $\begin{array}{c}\text { Ganho } \\
\text { peso }\end{array}$ & KT|V \\
\hline Câimbra & $0,748 \S$ & $0,210 ¥$ & $0,926 \S$ & $0,912 ¥$ & $0,653 ¥$ & $0,635 \S$ & $0,371 \S$ & $0,416 ¥$ & $0,806 ¥$ & $0,124 \S$ & $0,128 ¥$ & $0,343 ¥$ \\
\hline Hipotensão & $0,028 \S^{*}$ & 0,014¥* & $0,09 \S$ & $0,098 ¥$ & $0,054 ¥$ & $0,374 \S$ & $0,661 \S$ & $0,732 ¥$ & $0,832 ¥$ & $0,495 \S$ & 0,001¥* & $0,063 ¥$ \\
\hline Calafrios & $0,046 \S^{*}$ & $0,148 ¥$ & $0,856 \S$ & $0,932 ¥$ & $0,630 ¥$ & $0,276 \S$ & $0,667 \S$ & $0,714 ¥$ & $0,684 ¥$ & $0,393 \S$ & $0,026 ¥ *$ & $0,586 ¥$ \\
\hline Vômito & $0,005 \S^{*}$ & $0,720 ¥$ & $0,955 \S$ & $0,479 ¥$ & $0,185 ¥$ & $0,114 \S$ & $0,495 \S$ & $0,055 ¥$ & $0,300 ¥$ & $0,043 \S^{*}$ & $0,129 ¥$ & $0,020 ¥ *$ \\
\hline Cefaleia & $0,021 \S^{*}$ & $0,003 \#^{*}$ & $0,770 \S$ & $0,178 ¥$ & $0,401 ¥$ & $0,691 \S$ & $0,541 \S$ & $0,116 ¥$ & $0,357 ¥$ & $0,420 \S$ & $0,766 ¥$ & $0,361 ¥$ \\
\hline HA $\neq$ & $0,249 \S$ & 0,591 & $0,577 \S$ & $0,860 ¥$ & $0,378 ¥$ & $0,760 \S$ & $0,473 \S$ & $0,496 ¥$ & $0,625 ¥$ & $0,617 \S$ & $0,064 ¥$ & $0,431 ¥$ \\
\hline Arritmia & $0,203 \S$ & $0,012 ¥ *$ & $0,887 \S$ & 0,909¥ & $0,834 ¥$ & $0,395 \S$ & $0,756 \S$ & $0,618 ¥$ & $0,191 ¥$ & $0,278 \S$ & $0,762 ¥$ & $0,937 ¥$ \\
\hline Náuseas & 0,7419 & 0,109¥ & 0,7159 & $0,814 ¥$ & $0,256 ¥$ & 0,710 वा & 0,252 व & $0,476 ¥$ & $0,985 ¥$ & $0,584 \rrbracket$ & $0,661 ¥$ & $0,166 ¥$ \\
\hline Convulsões & 0,7419 & $0,743 ¥$ & 0,715 व & $0,356 ¥$ & $0,153 ¥$ & 0,290 व & 0,748 | & $0,476 ¥$ & $0,418 ¥$ & 0,416 I & $0,204 ¥$ & $0,519 ¥$ \\
\hline Agonia & 0,510 的 & $0,06 ¥$ & 0,465 I & $0,920 ¥$ & $0,100 ¥$ & 0,540 व & 0,865 I & $0,633 ¥$ & $0,790 ¥$ & 0,765 I & $0,890 ¥$ & $0,784 ¥$ \\
\hline Sudorese & $0,490 \rrbracket$ & $0,020 ¥$ & $0,465 \rrbracket$ & $0,520 ¥$ & $0,770 ¥$ & $0,540 \rrbracket$ & 0,1359 & $0,503 ¥$ & $0,440 ¥$ & $0,235 \rrbracket$ & $0,890 ¥$ & $0,221 ¥$ \\
\hline
\end{tabular}


Cogitare Enferm. (22)4: e52907, 2017

\begin{tabular}{|c|c|c|c|c|c|c|c|c|c|c|c|c|}
\hline Fraqueza & 0,510 व & $0,790 ¥$ & $0,535 \rrbracket$ & $0,520 ¥$ & $0,550 ¥$ & 0,460 व & 0,865 व & $0,503 ¥$ & $0,580 ¥$ & 0,765 I & $0,100 ¥$ & $0,784 ¥$ \\
\hline Falta de Ar & $0,490 \rrbracket$ & $0,740 ¥$ & $0,535 \rrbracket$ & $0,350 ¥$ & $0,770 ¥$ & 0,5409 & $0,865 \rrbracket$ & $0,191 ¥$ & $0,090 ¥$ & 0,235 व & $0,890 ¥$ & $0,784 ¥$ \\
\hline Dormência & 0,510 व & $0,390 ¥$ & 0,535 I & $0,520 ¥$ & $0,840 ¥$ & 0,540 ฯ & 0,8659 & $0,683 ¥$ & 0,930ғ & 0,765 व & $0,890 ¥$ & $0,784 ¥$ \\
\hline Tremores & 0,5109 & $0,090 ¥$ & $0,465 \rrbracket$ & $0,400 ¥$ & $0,550 ¥$ & 0,4609 & $0,865 \rrbracket$ & $0,081 ¥$ & $0,080 ¥$ & $0,235 \rrbracket$ & $0,890 ¥$ & $0,784 ¥$ \\
\hline
\end{tabular}

Legenda: †DRC - Doença Renal Crônica; łHA- Hipertensão Arterial; §Teste de Qui-Quadrado; 凤Teste exato de Fisher; ¥Teste de U de Mann-Whitney *Variáveis que apresentaram associação estatística significante.

As complicações e os fatores sociodemográficos e clínicos que apresentaram associações estatísticas foram: hipotensão e idade $(p=0,028)$, hipotensão e sexo ( $p=0014)$, hipotensão e ganho de peso interdialítico $(p=0,001)$; calafrios e sexo $(p=0,046)$, calafrios e ganho de peso interdialítico $(p=0,026)$; vômito e sexo $(p=0,005)$, vômito e sítio de diálise $(p=0,043)$, vômito e KT/V $(p=0,020)$; cefaleia e sexo $(p=0,021)$, cefaleia e idade $(p=0,003)$; tontura e anos de estudo $(p=0,018)$, tontura e religião $(p=0,048)$, tontura e ganho de peso interdialítico $(p=0,049)$; arritmia e idade $(p=0,012)$; diarreia e tempo de hemodiálise $(p=0,003)$; dor abdominal e ganho de peso interdialítico $(p=0,009)$; e sudorese e idade $(p=0,020)$.

\section{- DISCUSSÃO}

As complicações relacionadas à DRET e ao tratamento hemodialítico configuram-se como importantes fatores a serem observados e prevenidos, pois, dependendo de sua intensidade, podem culminar em consequências graves e menor qualidade de vida do paciente com DRET ${ }^{(8)}$.

A hipotensão foi uma complicação frequente na população estudada, diferindo da literatura ${ }^{(6)}$, a qual aborda essa como uma complicação secundária. Essa é uma resposta compensatória cardiovascular, que ocorre quando o ritmo da ultrafiltração ultrapassa a capacidade do preenchimento vascular ${ }^{(9)}$. A remoção rápida de substâncias osmoticamente ativas e o retardo do equilíbrio nos compartimentos intracelulares causam diminuição transitória na osmolalidade do plasma, justificando os episódios de hipotensão ${ }^{(10)}$.

Essa complicação associou-se à idade, ao sexo e ao ganho de peso interdialítico do paciente renal. Estudo $^{(11)}$ destaca a hipotensão como uma das principais complicações agudas durante o procedimento hemodialítico, identificando a sua prevalência em $50 \%$ de pacientes do sexo feminino submetidos à hemodiálise, com faixa-etária média de 47 anos, corroborando com os dados do presente estudo ${ }^{(11)}$. Quanto ao ganho de peso, pacientes com excesso de líquido estão mais propensos à redução da pressão arterial devido à maior remoção de líquido e de eletrólitos durante as sessões, principalmente se o volume de ultrafiltração for rápido ou excessivo ${ }^{(12)}$.

Nesse contexto, a clientela submetida à hemodiálise, em especial adultos do sexo feminino, que vivencia o ganho de peso em curto período, reflete aumento à vulnerabilidade para complicações como a hipotensão arterial. As associações identificadas remetem a atenção maior do enfermeiro, no sentido de promover educação em saúde para o seguimento adequado da dieta prescrita, principalmente à clientela dentro das características supracitadas, com vistas a evitar tal complicação.

Os calafrios geralmente estão associados a infecções no acesso vascular relacionadas a reações pirogênicas, desinfecção da máquina de hemodiálise e ao tratamento da água ${ }^{(10)}$. Estudo aponta para a ocorrência de calafrios relacionada à infecção em $60 \%$ dos entrevistados, indicando o início do tratamento com antibiótico desde o primeiro episódio dessa complicação ${ }^{(13)}$.

O calafrio apresentou associação estatística com as variáveis sexo e ganho de peso interdialítico, sendo o sexo feminino predominante na clientela investigada. Corroborando a relação dessa complicação com o sexo, estudo apontou para a ocorrência do calafrio em pacientes do sexo feminino, principalmente ${ }^{(14)}$. O calafrio remete-se a alterações musculoesqueléticas no paciente submetido à hemodiálise, as quais são manifestadas em maior proporção em mulheres ${ }^{(11)}$. 
Ao avaliar a associação entre essa complicação e o ganho de peso interdialítico, não foram encontrados estudos que demonstrassem de forma direta essa relação, porém estudo aponta que esse dado clínico é responsável por muitas das complicações durante a sessão de hemodiálise ${ }^{(14)}$. Nesse sentido, pacientes do sexo feminino que apresentam ganho de peso interdialítico estão mais vulneráveis à ocorrência de calafrios, fazendo-se necessário promover intervenções eficazes no sentido de evitar sua ocorrência, o que influencia sobremaneira na qualidade de vida do paciente dialítico.

A complicação vômito apresentou associação estatística com o sexo, sítio de diálise e KT/V. Os episódios de vômito durante a hemodiálise apresentam causas multifatoriais, como exemplo, o aumento na concentração de sódio e cálcio no dialisato e a gastroparesia ${ }^{(10)}$. As mulheres apresentaram maior severidade na ocorrência do vômito(15).

No que concerne à associação entre vômito e KT/V, não foram encontrados na literatura estudos que demonstrassem essa associação, entretanto, ressalta-se a relevância de se manter o tratamento hemodialítico em níveis adequados em conformidade ao estabelecido pelas diretrizes do National Kidney Foundation Disease Outcomes Quality Initiative, que recomendam que o valor do KT/V se mantenha maior que 1,2 indicando diálise adequada para reduzir a ocorrência de complicações durante as sessões ${ }^{(16)}$.

A cefaleia também foi elencada como uma complicação durante o período de hemodiálise, apresentando associação estatística com as variáveis sexo e idade. Autores relatam essa complicação como uma das mais frequentes entre a clientela hemodialítica ${ }^{(10)}$. Ela pode ocorrer frente à característica estressora do tratamento, além de sua ligação com níveis diminuídos de magnésio e níveis superiores de sódio nos períodos antes e após a diálise ${ }^{(17)}$.

Quanto ao sexo, na literatura ${ }^{(18)}$ a cefaleia ocorreu com predominância para o sexo masculino, não corroborando com a associação evidenciada na presente pesquisa. Quando associada com a idade, outro estudo evidenciou a cefaleia com umas das principais complicações hemodialíticas presentes em pacientes com idade mais avançada ${ }^{(19)}$.

Apesar dos dados divergentes, outra literatura evidencia a dor, em especial a cefaleia, como importante complicação aguda na hemodiálise, principalmente no sexo feminino, o qual se encontra mais vulnerável às alterações musculoesqueléticas dolorosas. Faz-se necessário ressaltar que a percepção da dor varia de acordo com cada indivíduo, e envolve uma gama de fatores influenciadores. Nesse sentido, uma avaliação holística quando a dor do paciente submetido à hemodiálise, em especial do sexo feminino, é relevante ${ }^{(11)}$.

A tontura apresentou associação estatística com anos de estudo, praticantes de alguma religião e ganho de peso interdialítico. A tontura ocorre em decorrência de episódios de hipotensão, explicandose pela retirada de líquido rotineira durante o tratamento, principalmente quando há um aumento excessivo no peso entre as sessões ${ }^{(20)}$.

Estudo envolvendo pacientes em hemodiálise evidenciou que $74,3 \%(n=26)$ dos entrevistados não tinham o ensino fundamental ${ }^{(21)}$, refletindo na dificuldade de assimilação da informação transmitida e consequentemente na pouca compreensão do tratamento e da doença, tornando-se um fator preponderante na redução da adesão ao tratamento ${ }^{(7)}$.

A relevância da religião pauta-se no dado de que pacientes hemodialítico praticantes de alguma religião apresentaram melhor qualidade de vida em relação àqueles que não apresentam crença religiosa $^{(22)}$. Outro estudo ${ }^{(23)}$, que analisou as atitudes religiosas de pessoas em tratamento hemodiálitico, apontou que pacientes que tinham menos conhecimento religioso tinham maior facilidade em aderir ao tratamento. Isso foi explicado pelo fato que o individuo religioso pode possuir uma ideia de proteção e ao se deparar com a mudança, suscitada pelo tratamento, pode provocar aversão e até mesmo interrupção do tratamento ${ }^{(23)}$, o que explicaria a associação encontrada na presente pesquisa entre praticante de religião e a complicação tontura.

A arritmia foi outra complicação presente nos pacientes, associando-se estatisticamente à idade. Estudo demonstra que pacientes em hemodiálise apresentam comumente problemas cardíacos, devido a mudanças importantes dos níveis de eletrólitos relacionados à atividade cardíaca. Essas mudanças podem afetar o processo de repolarização ${ }^{(24)}$. Quanto à idade, estudo demonstra que indivíduos 
portadores de DRET com idade avançada foram mais propensos a desenvolver arritmias ${ }^{(25)}$.

A diarreia apresentou associação estatística com o tempo de hemodiálise. Essa complicação pode refletir no agravamento do estado nutricional, prejudicando a absorção de alimentos ${ }^{(26)}$. Devido à imunossupressão, os pacientes em hemodiálise estão susceptíveis a infecções, incluindo a intestinal. Estudos relatam ainda associação estatística significante entre o tempo de diálise e a presença da diarreia nesses pacientes ${ }^{(26-27)}$.

Frente a isso, a dor abdominal também esteve presente e foi associada ao ganho de peso interdialítico. $\mathrm{O}$ excesso de peso interdialítico indica a necessidade de maiores retiradas de líquidos durante o procedimento hemodialítico, o que culmina em consequências agudas durante o procedimento de maior retirada.

Nesse sentido, a literatura aponta para alterações no estado nutricional do paciente influenciadas pelo ganho de peso interdialítico, em especial naqueles que apresentam maior tempo de procedimento hemodialítico, indicando a necessidade de se avaliar cada paciente em relação às complicações abdominais, regulando o peso interdialítico ${ }^{(28)}$.

Assim, intervenções relacionadas a pacientes em elevado tempo de tratamento hemodialítico, bem como aqueles com excesso de peso em curto período, devem ser traçadas, no sentido de reduzir a vulnerabilidade desses às alterações abdominais como a diarreia e a dor abdominal.

A sudorese é apresentada pela literatura como uma complicação durante a realização do procedimento $^{(29)}$. No presente estudo, associou-se estatisticamente com a idade. A literatura aponta para a necessidade de maior precisão na mensuração da transpiração em pacientes com hemodiálise, indicando a microvasculopatia como uma compliçção relacionada à duração da sessão de hemodiálise, o que altera a excreção e reabsorção do suor. Ademais, o volume de suor alterou de acordo com a idade, sendo mais prevalente naquela clientela acima de $50 \operatorname{anos}^{(30)}$.

As associações apresentadas neste trabalho podem auxiliar o profissional de saúde na identificação precoce das complicações du rante a hemodiálise, bem como a reconhecer os fatores sociodemográficos e clínicos que favorecem sua ocorrência, auxiliando-o a elencar ações precisas que reduzam os riscos e consequentemente a própria complicação, promovendo melhorias na qualidade de vida desse paciente.

As variáveis sociodemográficas: Estado civil, Renda, e Procedência, bem como a variável clínica Tempo de DRC não apresentaram associação estatística significante com as complicações vivenciadas durante o procedimento hemodialítico, entretanto, foram fundamentais para a caracterização da clientela.

Aponta-se, como limitação do presente estudo, o fato da identificação das complicações apenas nos pacientes submetidos à hemodiálise, não envolvendo outras terapias de substituição da função renal. Ademais, os achados do presente estudo suscitam o desenvolvimento de estudos longitudinais para comprovar as associações ora evidenciadas.

\section{CONCLUSÃO}

Conclui-se que as complicações vivenciadas pelo paciente renal submetido à hemodiálise podem sofrer influência dos aspectos sociodemográficos e clínicos do paciente. As associações estatísticas identificadas foram: hipotensão com idade, sexo e ganho de peso interdialítico; calafrios com sexo e ganho de peso interdialítico; vômito com sexo, sítio de diálise e KT/V; cefaleia com sexo e idade; tontura com anos de estudo, religião e ganho de peso interdialítico; arritmia e idade; diarreia e tempo de hemodiálise; dor abdominal e ganho de peso interdialítico.

Os resultados da pesquisa contribuem para o planejamento e execução dos cuidados aos pacientes em hemodiálise, resultando em ações pautadas nos aspectos sociais e clínicos vivenciados. A compreensão desses contribui para uma atuação em saúde precisa no intento de debelar complicações durante o procedimento hemodialítico, bem como na identificação precoce de vulnerabilidade nesta clientela. 
Ao Conselho Nacional de Ciência e Tecnologia (CNPq; projeto financiado processo 477559/2013-1). À Coordenação de Aperfeiçoamento de Pessoal de Nível Superior (CAPES).

\section{O REFERÊNCIAS}

1. National Kidney Foundation (KDIGO). Kidney disease improving global outcomes. Am. J. Kidney Dis. [Internet] 2013;3(1) [acesso em 14 jan 2017]. Disponível: https://www.theisn.org/kidney-disease-improving-globaloutcomes-kdigo.

2. Sesso RCC, Lopes AA, Thomé FS, Lugon JR, Watanabe Y, dos Santos DR. Diálise Crônica no Brasil: Relatório do Censo Brasileiro de Diálise, 2011. J Bras Nefrol. [Internet] 2012;34(3) [acesso em 14 jan 2017]. Disponível: http:// dx.doi.org/10.5935/0101-2800.20120009.

3. Mehmood Y, Ghafoor S, Ashraf MI, Riaz H, Atif SR, Saeed M. Intradialytic complications found in patients at a tertiary care hospital. Austin J Pharmacol Ther. [Internet] 2016;4(1) [acesso em 10 mar 2017]. Disponível: http:// austinpublishinggroup.com/pharmacology-therapeutics/fulltext/ajpt-v4-id1079.php.

4. Inrig JK, Molina C, D'Silva K, Kim C, Van Buren P, Allen JD, et al. Effect of low versus high dialysate sodium concentration on blood pressure and endothelial-derived vasoregulators during hemodialysis: a randomized crossover study. Am J Kidney Dis. [Internet] 2015;65(3) [acesso em 10 mar 2017]. Disponível: http://dx.doi. org/10.1053/j.ajkd.2014.10.021.

5. Goudarzian AH, Nia HS, Okamoto Y, Rhee CM, McFarlane P, Nejad FG. Adverse effects of hemodialysis on kidney patients: how good the evidence is. Int J MedInvest. [Internet] 2015;4(4) [acesso em 21 mar 2017]. Disponível: http://www.intjmi.com/browse.php?a_code=A-10-1-120\&slc_lang=en\&sid=1.

6. Coitinho D, Benetti ERR, Ubessi LD, Barbosa DA, Kirchner RM, Guido LA, et al. Intercorrências em hemodiálise e avaliação da saúde de pacientes renais crônicos. Av Enferm. [Internet] 2015;33(3) [acesso em 21 mar 2017]. Disponível http://dx.doi.org/10.15446/av.enferm.v33n3.38016.

7. Frazão CMFQ, Sá JDS, Paiva MGMN, Lira ALBC, Lopes MVO, Enders BC. Association Between Nursing Diagnoses and Socioeconomic/Clinical Characteristics of Patients on Hemodialysis. Int J Nurs Knowl. [Internet] 2015;26(3) [acesso em 4 dez 2016]. Disponível: http://dx.doi.org/10.1111/2047-3095.12051.

8. Kraus MA, Fluck JF, Weinhandl ED, Kansal S, Copland M, Komenda P, et al. A.Intensive hemodialysis and health-related quality of life. Am J Kidney Dis. [Internet] 2016;68(5) [acesso em 15 fev 2016]. Disponível: http:// dx.doi.org/10.1053/j.ajkd.2016.05.023.

9. Gil HW, Bang K, Lee SY, Han BG, Kim JK, Kim YO, et al. Efficacy of Hemocontrol biofeedback system in intradialytic hypotension-prone hemodialysis patients. J. Korean Med. Sci. [Internet] 2014;29(6) [acesso em 15 jan 2016]. Disponível: http://dx.doi.org/10.3346/jkms.2014.29.6.805.

10. Prabhakar SRG, Singh S, Rathore SS, Choudhary TA. Spectrum of intradialytic complications during hemodialysis and its management: A single-center experience. Saudi J Kidney Dis Transpl. [Internet] 2015;26(1) [acesso em 9 dez 2016]. Disponível: http://www.sjkdt.org/article.asp?issn=1319-2442;year=2015; volume=26;issue $=1$; spage $=168$; epage $=172$; aulast $=$ Prabhakar $\% 2 \mathrm{C}$.

11. Atik D, Karatepe H, Karatepe C, Demir S, Çınar S, Sökmen S. The effect of gender on the complications, pain intensity and pain management in hemodialysis patients. Int J Res Med Sci. [Internet] 2016;4(5) [acesso em 06 set 2017]. Disponível: http://www.scopemed.org/?mno=219666.

12. Morfin JA, Fluck RJ, WeinhandI ED, Kansal S, McCullough PA, Komenda P. Intensive Hemodialysis and Treatment Complications and Tolerability. Am J Kidney Dis. [Internet] 2016;68(5 Suppl 1) [acesso em 15 fev 2016]. Disponível: http://dx.doi.org/10.1053/j.ajkd.2016.05.021.

13. Shepshelovich D, Yelin D, Bach LO, Halevy N, Ziv Y, Green H, et al. Chills during hemodialysis: prediction and prevalence of bacterial infections - a retrospective cohort study. Am J Med. [Internet] 2017;130(4) [acesso em 10 mar 2017]. Disponível: http://dx.doi.org/10.1016/j.amjmed.2016.11.022. 
14. de Oliveira APC, de Sousa AS, de Mendonça AEO, da Silva RAR. Intradialytic complications in patients with chronic renal failure submitted to hemodialysis: integrative review. Rev enferm UFPE online. [Internet] 2013;7(11) [acesso em 14 jan 2016]. Disponível: http://www.revista.ufpe.br/revistaenfermagem/index.php/revista/article/ viewArticle/2547.

15. Asgari MR, Asghari F, Ghods AA, Ghorbani R, Motlagh NH, Rahaei F. Incidence and severity of nausea and vomiting in a group of maintenance hemodialysis patients. JRIP [Internet] 2017;6(1) [acesso em 10 mar 2017]. Disponível: http://dx.doi.org/10.15171/jrip.2017.09.

16. National Kidney Foundation (KDOQI). Clinical practice guideline for hemodialysis adequacy: 2015 update. Am J Kidney Dis. [Internet] 2015;66(5) [acesso em 06 set 2017]. Disponível: http://dx.doi.org/10.1053/j.ajkd.2015.07.015.

17. Levin M. Resident and Fellow Section. Headache. [Internet] 2009;49(6) [acesso em 4 dez 2016]. Disponível: http://dx.doi.org/10.1111/j.1526-4610.2009.01442.x

18. Stojimirovic B, Milinkovic M, Zidverc-Trajkovic J, Trbojevic-Stankovic J, Maric I, Milic M, et al. Dialysis headache in patients undergoing peritoneal dialysis and hemodialysis. Ren Fail. [Internet] 2015;37(2) [acesso em $10 \mathrm{dez}$ 2016]. Disponível: http://dx.doi.org/10.3109/0886022X.2014.982486.

19. Lakshman SG, Ravikumar P, Kar G, Das D, Bhattacharjee K, Bhattacharjee P. A comparative study of neurological complications in chronic kidney disease with special reference to its stages and haemodialysis status. J Clin Diagn Res. [Internet] 2016;10(12) [acesso em 14 jan 2017]. Disponível: http://dx.doi.org/10.7860/JCDR/2016/22815.8947.

20. Leung KCW, Quinn RR, Ravani P, MacRae JM. Ultrafiltration biofeedback guided by blood volume monitoring to reduce intradialytic hypotensive episodes in hemodialysis: study protocol for a randomized controlled trial. Trials. [Internet] 2014;(15) [acesso em 14 dez 2016]. Disponível: http://dx.doi.org /10.1186/1745-6215-15-483.

\section{Teixeira ALF, Ubessi LD, Benetti ERR, Kirchner RM, Barbosa DA, Stumm EMF.}

Events associated with the daily life of elderly people on hemodialysis. Ciênc. cuid. Saúde [Internet] 2015;14(3) [acesso em 14 fev 2017]. Disponível: http://dx.doi.org/10.4025/cienccuidsaude.v14i3.23003.

22. Fukushima RLM, Menezes ALC, Inouye K, Pavarini SCI, Orlandi FS. Fatores associados à qualidade de vida de pacientes renais crônicos em hemodiálise. Acta paul. enferm. [Internet] 2016;29(5) [acesso em 07 set 2017]. Disponível: http://dx.doi.org/10.1590/1982-0194201600072.

23. Almeida KCS, de Sousa MNA, de Oliveira T, Bezerra ALD, Nunes RMV, de Medeiros RC. Atitude religiosa de pessoas com doença renal crônica em tratamento hemodialítico. Rev Enferm UFPI. [Internet] 2016;5(2) [acesso em 06 set 2017]. Disponível: http://www.ojs.ufpi.br/index.php/reufpi/article/view/5042.

24. Poulikakos D, Banerjee D, Malik M. Major arrhythmic events and T wave morphology descriptors in hemodialyzed patients. J. Eletrocardiol. [Internet] 2014;47(2) [acesso em 17 dez 2016]. Disponível: https://doi. org/10.1016/j.jelectrocard.2013.11.010.

25. Bonato FOB, Lemos MM, Cassiolato JL, Canziani MEF. Prevalence of ventricular arrhythmia and its associated factors in Nondialyzed Chronic Kidney Disease Patients. PLoS ONE. [Internet] 2013;8(6) [acesso 7 dez 2016]. Disponível: http://doi.org/10.1371/journal.pone.0066036.

26. Silva LF, Lopes GB, Matos CM, Brito KQ, Amoedo MK, Azevedo MF, et al. Gastrointestinal symptoms and nutritional status in women and men on maintenance hemodialysis. J Ren Nutr. [Internet] 2012;22(3) [acesso em 17 dez 2016]. Disponível: http://dx.doi.org/10.1053/j.jrn.2011.07.007.

27. Gil FF, Barros MJ, Macedo NA, Júnior CGE, Redoan R, Busatti H, et al. Prevalence of intestinal parasitism and associated symptomatology among hemodialysis patients. Rev. Inst. Med. trop. S. Paulo. [Internet] 2013;55(2) [acesso em 17 dez 2016]. Disponível: http://dx.doi.org/10.1590/S0036-46652013000200001.

28. Ferraz SF, Freitas ATVS, Vaz IMF, Campos MIVAM, Peixoto MRG, Pereira ERS. Estado nutricional e ganho de peso interdialítico de pacientes com doença renal crônica em hemodiálise. J. Bras. Nefrol. [Internet] 2015;37(3) [acesso em 07 set 2017]. Disponível: http://dx.doi.org/10.5935/0101-2800.20150050.

29. Silva MS, Marini TSO, da Silva CFB. Enfermagem e suas intervenções nas principais complicações ocorridas durante a sessão de hemodiálise. Rev. Enfermagem e Saúde Coletiva. [Internet] 2016;1(2) [acesso em 25 set 2017]. Disponível http://revesc.org/index.php/revesc/article/view/7. 
30. Murota H. Sweating in systemic abnormalities: Uremia and Diabetes Mellitus. Curr Probl Dermatol. [Internet] 2016;51 [acesso em 25 set 2017]. Disponível: http://dx.doi.org/10.1159/000446760. 